\title{
INDUCTANCE MODELING FOR ON-CHIP INTERCONNECTS
}

\author{
Shang-Wei Tu*, Wen-Zen Shen*, Yao-Wen Chang**, and Tai-Chen Chen** \\ *Department of Electronics Engineering, National Chiao Tung University, Hsinchu, Taiwan ROC \\ **Graduate Institute of Electronics Engineering \& Department of Electrical Engineering, National Taiwan University, Taipei, Taiwan ROC
}

\begin{abstract}
As the operation frequency reaches gigahertz in deep-submicron designs, the effects of inductance on noise and delay can no longer be neglected. Most of the previous works on inductance extraction are fieldsolvers, which are intrinsically more accurate but computationally expensive. Others focus on modeling the inductances of special routing topologies such as the bus structure. Therefore, it is not suitable to incorporate them on-line into a layout (placement and routing) tool for inductance (delay and noise) optimization. In this paper, we consider the overlapping of unequal wire lengths and dimensions to efficiently extract the loop inductance from the coplanar interconnect structure. The difference between our simulation results and the estimation values obtained by FastHenry [12] is within 10\% for practical cases. In particular, our method is very fast. Based on our study, we also suggest several routing topologies for inductance minimization.
\end{abstract}

\section{INTRODUCTION}

As technology advances into the very deep-submicron era, interconnection delay dominates overall circuit performance and noise becomes more serious than before. Therefore, accurately predicting the interconnection delay and noise becomes a major challenge in high performance designs. For deep-submicron, high-performance circuits, ignoring inductance effects may incur a large amount of error, since an $R C$ model as compared to an $R L C$ model may create errors of up to $30 \%$ in the total propagation delay of a repeater system [11], and in some worst cases the noise coupling due to $\mathrm{C}$ and $\mathrm{L}$ may reach around $55 \%$ of the supply voltage [9]. As technology improves and die size increases, short rise/fall times of signals and long wires make inductance effects much more significant than before [14]. Therefore, it is very importance to consider the self and coupling inductance.

Inductance extraction has been studied extensively in the literature. Grover [8] collected many formulas for extracting inductance. However, as mentioned in [10], there are limitations of applying the equations. For example, they do not consider internal inductance, and wire widths are not considered for mutual inductance. Greengard developed a multipole method to extract inductances [7]. Phillips, Kamon, and White [13] proposed an FFT-based approach for 3-D inductance extraction. Beattie and Pileggi proposed a hierarchical refinement method [3] that improves the running time for capturing the near field effects. Also, Beattie et al. proposed equipotential shells methodology [2] to efficiently extract the inductance of the bus structure. He et al. presented a table-based approach to identify the structure of a circuit for inductance extraction [10].

Most of the previous works are field-solvers that are intrinsically more accurate but are computationally expensive. Others focus on modeling the inductances of special routing topologies such as the bus structure. Therefore, it is not suitable to incorporate them on-line into a layout tool for inductance optimization. As [5] pointed out, no good approximation formula exists for coupling inductances of two parallel lines of unequal lengths and dimensions. We consider the overlapping of unequal wire lengths and dimensions (widths and heights) to extract the self and coupling inductance. We derive formulae and models to approximate both self and coupling inductance efficiently yet accurately for the coplanar routing structure. Simulation results show that the difference of the inductance values extracted from our models for practical cases is within $10 \%$ for the wires of unequal lengths and dimensions, compared with
FastHenry [12]. In particular, our method is extremely fast. For example, the running time for extracting the coupling inductance of two wires of 2000 um by using our analytical formulas is typically within 0.11 seconds using Mathematica on a $566 \mathrm{MHz}$ Cerelon PC with $128 \mathrm{MB}$ RAM while FastHenry requires 141 seconds on SUN Sparc Ultra 60 with dual CPUs and 2GB memory. Therefore, it is feasible to incorporate our model into a layout tool on-line.

Recently, researchers presented layout structures to reduce inductance such as staggered inverter [4] and twisted-bundle layout structure [15]. Based on our inductance modeling, we also suggest good routing topologies for inductance reduction. Simulation results justify our findings.

The rest of this paper is organized as follows. Section 2 describes the coplanar interconnect structure and some basic electromagnetic concepts. Section 3 derives formulae for self and coupling inductance. Section 4 proposes some routing topologies for inductance minimization. Section 5 gives the simulation results on the comparisons between our methods and FastHenry. Finally, Section 6 concludes our work.

\section{PRELIMINARIES}

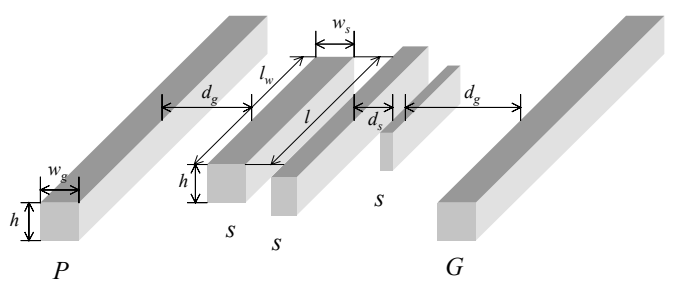

Figure 1: Coplanar interconnect structure.

Figure 1 depicts the coplanar interconnect structure. Here, $s$ stands for a signal wire while $P$ and $G$ stands for a power and a ground grid of identical width, $w_{g}$. Throughout this paper, both $P$ and $G$ are considered as ground wires. Assume that all wires are of the same height $h$, the width and length of each signal wire are $w_{s}$ and $l_{w}$ respectively, and the overlapping length of two signal wires is $l$. Throughout this paper, we assume quasi-static conditions [1].

Before deriving the inductance for a wire, we shall first determine the magnetic flux density of the wire carrying current $I$ by using Ampère's circuital law [6]:

$$
\oint_{C} \vec{B} \cdot d \vec{l}=\mu_{0} I,
$$

where $\boldsymbol{B}$ is magnetic flux density, the path $C$ for the line integral is the contour bounding the surface $S, I$ is the total current through $S$, and $\mu_{0}$ is permeability whose value is $4 \pi \times 10^{-7}(\mathrm{H} / \mathrm{m})$.

Then we derive the self-inductance $\left(L_{i}\right)$ and coupling inductance $\left(L_{i j}\right)$ as in Equations (2) and (3), respectively, by using Faraday's law [6]:

$$
\begin{gathered}
L_{i}=\frac{\Lambda_{i}}{I_{i}}=\frac{N_{i} \Phi_{i}}{I_{i}}=\frac{N_{i}}{I_{i}} \int_{S_{i}} \vec{B}_{i} \cdot d \vec{s}_{i}, \\
L_{i j}=\frac{\Lambda_{i j}}{I_{i}}=\frac{N_{j} \Phi_{i j}}{I_{i}}=\frac{N_{j}}{I_{i}} \int_{S_{j}} \vec{B}_{i} \cdot d \vec{s}_{j} .
\end{gathered}
$$

In Equation (2), $\Lambda_{\mathrm{i}}$ is flux linkage, $\Phi_{\mathrm{i}}$ is flux, $S_{i}$ represents the surface bounded by the loop $C$ of wire $i, N_{i}$ is the number of turns of loop $C$, and $\boldsymbol{B}_{i}$ is the magnetic flux density due to current $I_{i}$ in wire $i$. In Equation (3), $S_{j}$ is the surface bounded by the loop $C_{j}$ of wire $j, N_{j}$ is the number of 
turns of loop $C_{j}$, and $\boldsymbol{B}_{i}$ is the magnetic flux density due to current $I_{i}$ in wire $i$.

Same as [15], we assume that all signals use the nearest $P / G$ wires as their return paths, and other wires that are not involved in the computation for the inductance between wires $i$ and $j$ are floating.

\section{INDUCTANCE MODELING}

\subsection{Self-inductance}

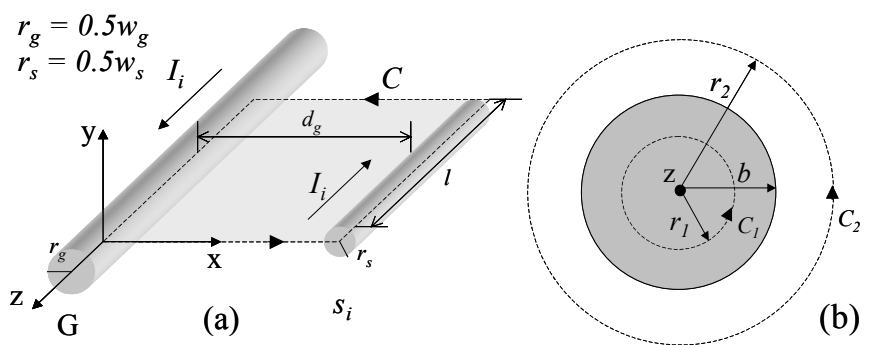

Figure 2: (a) Degeneration of metal wire from Figure 1. (b) Cross-section view of circular conductor.

Because the rectangular shape of wires, as illustrated in Figure 1, complicates the computation of magnetic flux density, we apply circular conductors to approximate the results, as illustrated in Figure 2(a). If the current $I_{i}$ in wire $s_{i}$ flows as the direction shown in Figure 2(a), the current loop $C$ can be determined as the dashed line. The area of $S_{i}$, which is the gray region in Figure 2, is determined by the enclosed region of the loop $C$. To derive self-inductance from Equation (1), we must know $N_{i}, S_{i}$, and $\boldsymbol{B}_{i}$. Although $N_{i}=1$ and $S_{i}$ are already known, $\boldsymbol{B}_{\boldsymbol{i}}$ is still unknown. Hence, we shall derive the formula of $\boldsymbol{B}_{\boldsymbol{i}}$ first. Here, the unit of $L^{\prime}$ is henry per meter $(\mathrm{H} / \mathrm{m})$ and the unit of $L$ is henry $(\mathrm{H})$.

Figure 2(b) depicts the cross-sectional view of a circular conductor whose radius is $b$. If we align the conductor's center along the $z$-axis in the cylindrical coordination system, current $I_{i}$ flows in the $z$ direction. Assume that current $I_{i}$ is uniformly distributed in the conductor, and the conductor is sufficiently long $\left(l_{w}>>r\right)$, we can derive Equations (4) and (5) by Equation (1) as follows:

$$
\begin{gathered}
\vec{B}_{\text {in }}=\hat{a}_{\phi} \frac{\mu_{0} r_{1} I_{i}}{2 \pi b^{2}}, \quad r_{1} \leq b \\
\vec{B}_{\text {out }}=\hat{a}_{\phi} \frac{\mu_{0} I_{i}}{2 \pi r_{2}}, \quad r_{2} \geq b
\end{gathered}
$$

where $\boldsymbol{B}_{\text {in }}$ is the magnetic flux density inside the conductor and $\boldsymbol{B}_{\text {out }}$ is the magnetic flux density outside the conductor.

We derive the formula of the internal inductance per unit length for a circular wire. Consider an annular ring in the inner conductor between radius $r$ and $r+d r(r \leq b)$. The current in a unit length of this annular ring is linked by the flux that can be obtained from Equation (4), we have

$$
d \Phi_{\text {in }}^{\prime}=\int_{r}^{b} B_{\text {in }} d r=\frac{\mu_{0} I_{i}}{4 \pi b^{2}}\left(b^{2}-r^{2}\right) \cdot
$$

The current in the annular ring, however, is only a fraction ( $\left.2 \pi r d r / \pi b^{2}=2 r d r / b^{2}\right)$ of the total current $I_{i}$. Hence, we can obtain the unit-length internal inductance of the circular wire by using Equations (2) and (6):

$$
L_{i n}^{\prime}=\frac{\Lambda_{i n}^{\prime}}{I_{i}}=\frac{\int_{0}^{b} d \Lambda_{i n}^{\prime}}{I_{i}}=\frac{\int_{0}^{b} 2 r d r d \Phi_{i n}^{\prime}}{b^{2} I_{i}}=\frac{\mu_{0}}{8 \pi} .
$$

Then we derive the external self-inductance. In the $x z$-plane where the two wires lie, as in Figure 2, the contributing $\boldsymbol{B}$ vectors due to the equal and opposite currents in the two wires have only a $y$-component. Hence, from Equation (5), we have

$$
\begin{gathered}
B_{G}=\frac{\mu_{0} I_{i}}{2 \pi x}, \\
B_{s_{i}}=\frac{\mu_{0} I_{i}}{2 \pi\left(d_{g}+r_{g}+r_{s}-x\right)} .
\end{gathered}
$$

Therefore, the unit-length external inductance of Figure 2 is
$L_{e x}^{\prime}=\frac{\Phi_{e x}^{\prime}}{I_{i}}=\frac{\int_{r_{g}}^{r_{g}+d_{g}}\left(B_{G}+B_{s_{i}}\right) d x}{I_{i}}=\frac{\mu_{0}}{2 \pi} \ln \left(\frac{\left(r_{g}+d_{g}\right)\left(r_{s}+d_{g}\right)}{r_{s} r_{g}}\right) \cdot(10)$

Finally, we can derive the total self-inductance $\left(L_{i}\right)$ of Figure 2 from Equations (7), (10), and the signal wire length $l_{w}$ as follows:

$L_{i}=l_{w}\left(2 L_{i n}^{\prime}+L_{e x}^{\prime}\right)=l_{w}\left(\frac{\mu_{0}}{4 \pi}+\frac{\mu_{0}}{2 \pi} \ln \left(\frac{\left(r_{g}+d_{g}\right)\left(r_{s}+d_{g}\right)}{r_{s} r_{g}}\right)\right)$

If another ground wire exists on the other side of the signal wire separated by the distance $d_{g 2}\left(d_{g 2} \geq d_{g}\right)$, the current flowing in the nearest $G$ wire will decrease. We modify Equation (11) to Equation (12) with the branch current of $0.5 I_{i}$ in the $G$ wire, which is given by the Kirchhoff's Current law.

$$
L_{i}=\frac{l_{w} \mu_{0}}{2 \pi}\left(0.375+\ln \left(\frac{\left(r_{g}+d_{g}\right)^{0.5}\left(r_{s}+d_{g}\right)}{r_{s} r_{g}^{0.5}}\right)\right)
$$

If there are $n$ ground wires, we can simplify these cases to the two ground wires system. We named the above formula $A M A S$ (Analytical $\underline{M}$ odel $\underline{A}$ pproximation of $\underline{S}$ elf-inductance).

\subsection{Coupling Inductance}

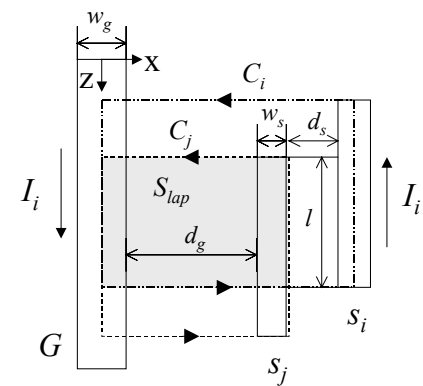

Figure 3: Top view of the coplanar structure.

As in the previous subsection, we consider single ground wire system first. The top view of the coplanar structure is shown in Figure 3. Now, we consider the $x z$-plane where the three wires lie. The $\boldsymbol{B}$ vectors due to the currents in $G$ and $s_{i}$ wires have only a y-component. From Equation (5), we have

$$
\begin{aligned}
& B_{G}=\frac{\mu_{0} I_{i}}{2 \pi x} \\
& B_{s_{i}}=\frac{\mu_{0} I_{i}}{2 \pi\left(0.5 w_{g}+d_{g}+d_{s}+1.5 w_{s}-x\right)} .
\end{aligned}
$$

The coupling inductance $L_{i j}$ is proportional to the overlapping area of $S_{i}$ and $S_{j}$. The overlapping area $S_{\text {lap }}$ is shown as the gray region in Figure 3. We can derive the external inductance per unit length from Equation (3) as follows:

$$
\begin{aligned}
& L_{e x}^{\prime}=\frac{\Lambda_{e x}^{\prime}}{I_{i}}=\frac{\Phi_{e x}^{\prime}}{I_{i}}=\frac{1}{l I_{i}} \int_{S_{l a p}} B d s_{l a p}=\int_{0.5 w_{g}}^{0.5 w_{g}+d_{g}+w_{s}}\left(B_{G}+B_{s_{i}}\right) d x \\
& =\frac{\mu_{0}}{2 \pi} \ln \left(\frac{\left(0.5 w_{g}+d_{g}+w_{s}\right)\left(d_{g}+d_{s}+1.5 w_{s}\right)}{0.5 w_{g}\left(d_{s}+0.5 w_{s}\right)}\right) .
\end{aligned}
$$

The internal inductance due to the $\mathrm{G}$ wire is the same as that of Equation (7). Hence, the total coupling inductance shown in Figure 3 is

$$
\begin{aligned}
& L_{i j}=L_{j i}=l\left(L_{i n}^{\prime}+L_{e x}^{\prime}\right) \\
& =\frac{l \mu_{0}}{2 \pi}\left(\frac{1}{4}+\ln \left(\frac{\left(0.5 w_{g}+d_{g}+w_{s}\right)\left(d_{g}+d_{s}+1.5 w_{s}\right)}{0.5 w_{g}\left(d_{s}+0.5 w_{s}\right)}\right)\right) .
\end{aligned}
$$

If another ground wire exists on the other side of the signal wire at distance $d_{g 2}\left(d_{g 2} \geq d_{g}+d_{s}+w_{s}\right)$, the current flowing in the nearest $G$ wire will decrease. Again, we can modify Equation (16) to (17) with the branch current of $0.5 I_{i}$ in the $G$ wire as follows:

$L_{i j}=L_{j i}=\frac{l \mu_{0}}{2 \pi}\left(\frac{1}{8}+\ln \left(\frac{\left(0.5 w_{g}+d_{g}+w_{s}\right)^{0.5}\left(d_{g}+d_{s}+1.5 w_{s}\right)}{\left(0.5 w_{g}\right)^{0.5}\left(d_{s}+0.5 w_{s}\right)}\right)\right)$ (17) 
If there are $n$ ground wires, we can simplify these cases to the two ground wires system. We name the above formula $A M A C$ (Analytical $\underline{M}$ odel $\underline{A}$ pproximation of $\underline{C}$ oupling Inductance).

\subsection{Modeling Short Wires and Small Coupling}

Previous subsections are all based on the assumption that wires are sufficiently long, namely, $l_{w}>d_{g}$. When wire lengths are comparable to wire spaces, however, Equation (5) may no longer be accurate. Further, coupling inductance still exists even if two signal wires are not overlapped. Therefore, the equations derived in Sections 3.1 and 3.2 may cause significant errors in some corner cases. To cope with these problems, we should use more sophisticated formulas to approximate the inductance for the cases when (a) the wire length is comparable to its distance to the nearest ground wire, and (b) the overlapping length is much smaller than the signal wire length.
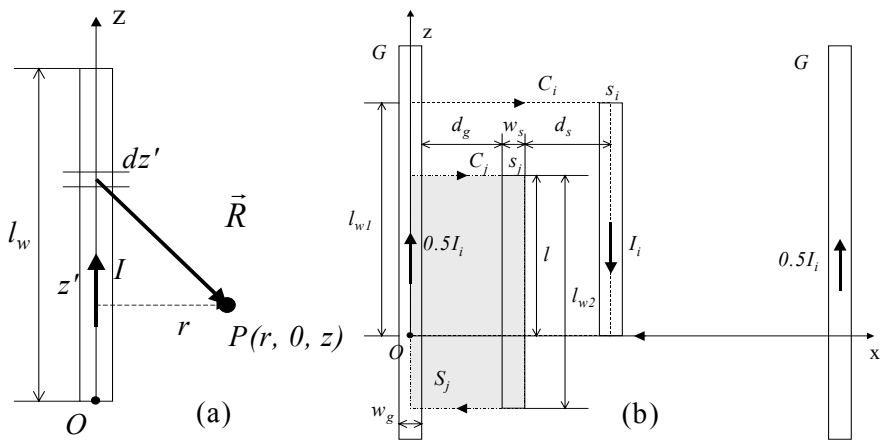

Figure 4: (a) Top view of a carrying current wire. (b) Top view of the two-ground wires system.

Equation (18), the Biot-Savart law, gives the magnetic flux density for condition (a) or (b):

$$
\vec{B}=\oint_{C^{\prime}} d \vec{B}=\frac{\mu_{0} I}{4 \pi} \oint_{C^{\prime}} \frac{d \vec{l}^{\prime} \times \vec{R}}{R^{3}}
$$

where $C^{\prime}$ is the closed path of current $I$, and $\vec{R}$ is the vector directed from the source to the field point. If we have a line segment carrying current $I$ along the $z$-axis in the cylinder coordinate shown in Figure 4(a). The resulting $\boldsymbol{B}$ vector is

$$
\vec{B}=\hat{a}_{\phi} \frac{\mu_{0} I}{4 \pi} \int_{0}^{l_{w}} \frac{r d z^{\prime}}{\left(r^{2}+\left(z-z^{\prime}\right)^{2}\right)^{3 / 2}} .
$$

Next, we consider the coplanar structure of Figure 4(b). From Equation (19), we know the contributing $\mathbf{B}$ vectors due to $s_{i}$ and $G$ wires of Figure 4(b). The flux linkages due to $s_{i}$ and $G$ wires are

$$
\begin{gathered}
\Lambda_{G}=\Phi_{G}=\int_{S_{j}} B_{G} d s_{j}=\int_{0.5 w_{g}}^{0.5 w_{g}+d_{g}+w_{s}} \int_{l-l_{w}}^{l} B_{G} d z d x, \\
\Lambda_{s_{i}}=\Phi_{s_{i}}=\int_{S_{j}} B_{s_{i}} d s_{j}=\int_{0.5 w_{g}}^{0.5 w_{g}+d_{g}+w_{s}} \int_{l-l_{w}}^{l} B_{s_{i}} d z d x .
\end{gathered}
$$

Therefore, the total external inductance is

$$
L_{e x}=\Lambda_{e x} / I_{i}=\left(\Lambda_{G}+\Lambda_{s_{i}}\right) / \mathrm{I}_{\mathrm{i}} .
$$

Hence, the total coupling inductance is

$$
L_{i j}=L_{i n}+L_{e x}=0.5 l \frac{\mu_{0}}{8 \pi}+L_{e x} .
$$

We name the above formula IAMAC (Improved Analytical Model Approximation of Coupling Inductance).

Next, we derive the formula of the self-inductance for the situation when a signal wire length is comparable to its distance to the nearest ground wire. Considering the two ground wires system of Figure 4(b), the internal inductance is

$$
L_{\text {in }}=(1+0.5) \times l_{w} \times \frac{\mu_{0}}{8 \pi} .
$$

By Equation (2), we have

$$
\begin{aligned}
& \Lambda_{G}=\Phi_{G}=\int_{S_{j}} B_{G} d s_{j}=\int_{0.5 w_{g}}^{0.5 w_{g}+d_{g}} \int_{l-l_{w}}^{l} B_{G} d z d x \\
& \Lambda_{s_{i}}=\Phi_{s_{i}}=\int_{S_{j}} B_{s_{i}} d s_{j}=\int_{0.5 w_{s}}^{0.5 w_{s}+d_{g}} \int_{l-l_{w}}^{l} B_{s_{i}} d z d x .
\end{aligned}
$$

$\mathrm{B}_{\mathrm{G}}$ and $\mathrm{B}_{\mathrm{si}}$ are known from Equation (19), and the total self-inductance is

$$
L_{i}=L_{i n}+L_{e x}=1.5 l_{w} \frac{\mu_{0}}{8 \pi}+L_{e x} .
$$

We name the above formula IAMAS (Improved Analytical Model Approximation of $\underline{S}$ elf-inductance).

\section{LAYOUT IMPLICATIONS}

In this section, we suggest some better routing topologies to reduce coupling inductance. As an example, if there are three terminals-one source and two sinks as shown in Figure 5-to be routed from the source $A$ to two sinks $B$ and $C$, which one in Figure 6 is the best routing topology to reduce the coupling inductance? We used FastHenry to extract the coupling inductance of each routing topology and obtained the results listed in Table 1.
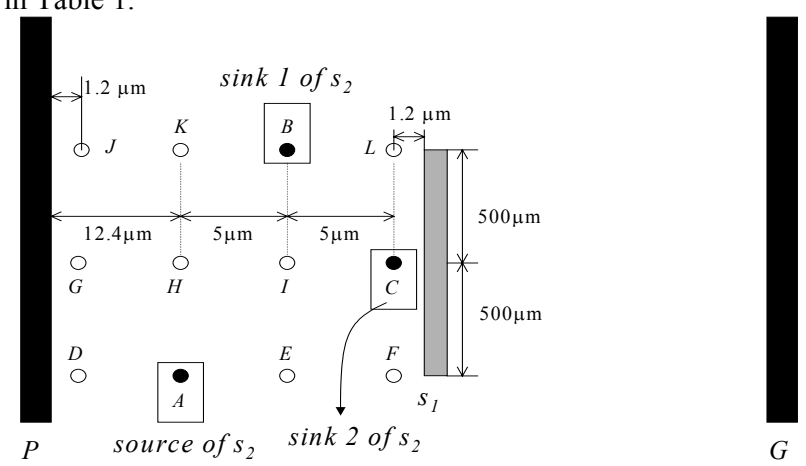

Figure 5: $s_{1}$ stands for signal wire $1 ; s_{2}$ stands for signal wire $2 ; A$ stands for the source of $s_{2} ; B$ and $C$ stand for the two sink of $s_{2} ; E, F, G, H, I, J$, and $K$ are Steiner points which may be used for routing.

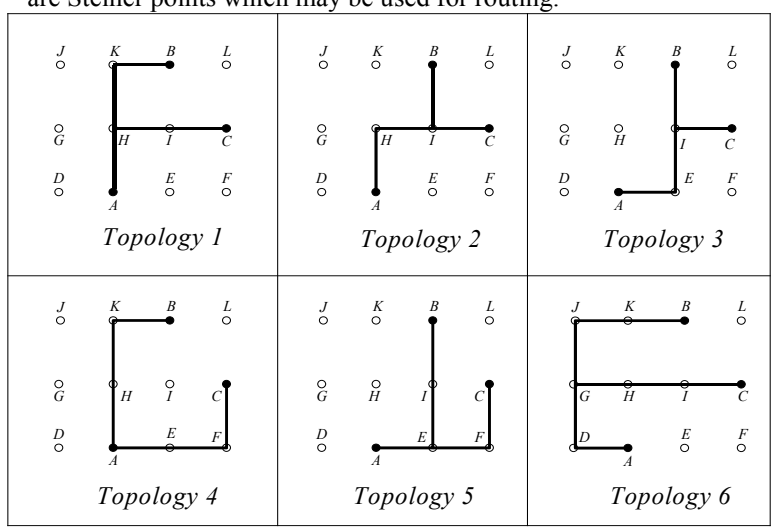

Figure 6:Potential routing topologies of Figure 5 (source: $A$ ).

From Equation (3), $S_{j}$ and $\boldsymbol{B}_{\boldsymbol{i}}$ are the two major factors in determining the value of coupling inductance. As shown in Table 1, Topology 6 results in the smallest coupling inductance because it has the smallest $S_{2}$ among the six routing topologies. Therefore, Topology 6 has the smallest $L_{12}$ because the $\boldsymbol{B}_{1}$ from $s_{1}$ is identical to all topologies. Considering the optimization of coupling inductance in this example, Topology 6 is the best choice even though it has the largest wire length.

\begin{tabular}{|c|r|c|}
\hline Topology & $\begin{array}{c}\text { Coupling inductance } \\
\text { extracted by FastHenry }\end{array}$ & $\begin{array}{c}\text { Coupling inductance ranking } \\
\text { (from low to high) }\end{array}$ \\
\hline 1 & $2.82042 \times 10^{-10} \mathrm{H}$ & 2 \\
\hline 2 & $2.82579 \times 10^{-10} \mathrm{H}$ & 3 \\
\hline 3 & $3.64932 \times 10^{-10} \mathrm{H}$ & 4 \\
\hline 4 & $5.21743 \times 10^{-10} \mathrm{H}$ & 5 \\
\hline 5 & $5.64098 \times 10^{-10} \mathrm{H}$ & 6 \\
\hline 6 & $0.48866 \times 10^{-10} \mathrm{H}$ & 1 \\
\hline \multicolumn{3}{|c|}{ Table 1: Coupling inductance of the routing topologies in Figure 6. } \\
\hline
\end{tabular}

We also consider the effect of the assignment for the source pin on inductance: Will the coupling inductance be different if we change the source? For Topology 6, the results are shown in Table 2 for assigning A, $\mathrm{B}$, or $\mathrm{C}$ as the source of the net.

\begin{tabular}{l|l|l|} 
Source in & Coupling inductance & Coupling inductance ranking
\end{tabular}

\begin{tabular}{ll|l} 
Topology 6 & extracted by FastHenry & (from low to high)
\end{tabular} 


\begin{tabular}{|c|c|c|}
\hline$A$ & $0.488662 \times 10^{-10} \mathrm{H}$ & 2 \\
\hline$B$ & $0.480616 \times 10^{-10} \mathrm{H}$ & 3 \\
\hline$C$ & $0.008044 \times 10^{-10} \mathrm{H}$ & 1 \\
\hline
\end{tabular}

Table 2: Results of coupling inductances for different source assignments in Topology 6.

From Table 2, making $C$ the source pin results in the smallest coupling inductance. This phenomenon is due to the fact when current flows from $C$ to $A$ and $B$, the currents in line segments $\overline{G J}$ and $\overline{G D}$ of Topology 6 result in the magnetic fluxes of opposite directions. Therefore, source-pin assignment is also an important issue for coupling-inductance optimization, for which we suggest that the source pin be assigned to the pin in the middle.

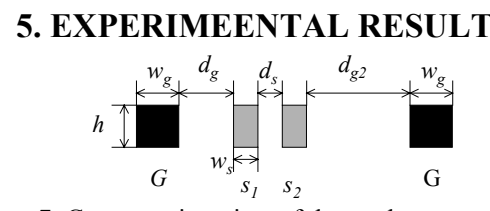

Figure 7: Cross-section view of the coplanar structure.

We conducted the following experiments: (1) the accuracy of AMAS and IAMAS for self-inductance extraction, and (2) the accuracy of AMAC and IAMAC for coupling-inductance extraction.

In the first experiment, we tested the accuracy of AMAS and IAMAS for self-inductance extraction based on the structure of Figure 7 with various $1_{\mathrm{w}}$ 's (signal wire lengths), $\mathrm{d}_{\mathrm{g}}, \mathrm{w}_{\mathrm{s}}, \mathrm{w}_{\mathrm{g}}$. Also, we set $\mathrm{d}_{\mathrm{g} 2}=\mathrm{d}_{\mathrm{g}}=18.4$ $\mu \mathrm{m}$ and $\mathrm{h}=2 \mu \mathrm{m}$. The length of the $\mathrm{G}$ wire was $4500 \mu \mathrm{m}$. The experimental results are given in Table 3, for which FastHenry was run on a SUN Ultra 60 model 1450 with dual CPUs and 2GB RAM and AMAS and IAMAS on a $566 \mathrm{MHz}$ Cerelon PC with $128 \mathrm{MB}$ RAM by using Mathematica Version 3.0. Identical values of parameters and conditions were applied to the second experiments on the coupling inductance whose results are shown in Table 4. Compared with FastHenry, the average errors of our modeling are within $10 \%$, except for the two corner cases: (1) the wire length is comparable to its distance to the nearest ground wire, and (2) the overlapping length is much smaller than the signal wire length. This phenomenon justifies our observation in Section 3.3. For these corner cases, our improved formulae can achieve significantly more accurate estimations than the original ones, as shown in Tables 3 and 4. The results show the accuracy of our work. In particular, our method is extremely fast. For example, the running time for extracting the coupling inductance of two wires of 2000 um by using our analytical formulas is typically within 0.11 seconds using Mathematica on a $566 \mathrm{MHz}$ Cerelon PC with 128 MB RAM while FastHenry requires 141 seconds on SUN
Sparc Ultra 60 with dual CPUs and 2GB memory. Therefore, it is feasible to incorporate our model into a layout tool on-line.

\section{CONCULSION}

We have derived analytical formulae to efficiently approximate the self and coupling inductance for wires of unequal lengths and dimensions. Experimental results have shown the accuracy of our formulae. In particular, our modeling is extremely efficient, and thus can be incorporated into a layout tool for inductance optimization. Based on our studies, we also have suggested some good routing topologies of signal wires to reduce the coupling inductance. Our findings should be very useful for layout designs with inductance considerations.

\section{REFERENCES}

[1] M. W. Beattie and L. T. Pileggi, "Inductance 101: Modeling and Extraction," Design Automation Conference, pp. 323-328, 2001.

[2] M. W. Beattie, B. Krauter, L. Alatan, and L. Pileggi, "Equipotential Shells for Efficient Inductance Extraction," IEEE Trans. Computer-Aided Design, Vol. 20, Issue: 1, pp. 70-79, January 2001.

[3] M. W. Beattie and L. T. Pileggi, "Electromagnetic Parasitic Extraction via a Multipole Method with Hierarchical Refinement," International Conference on Computer Added Design, pp. 437-444, 1999.

[4] M. W. Beattie and L. T. Pileggi, "IC analyses including extracted inductance models," Proceedings of $36^{\text {th }}$ Design Automation Conference, pp. 915-920, 1999.

[5] C. K. Cheng, J. Lillis, S. Lin, and N. Chang, Interconnect Analysis and Synthesis, John Wiley and Sons Inc, 2000

[6] D. K. Cheng, Filed and Wave Electromagnetics, $2^{\text {nd }}$ Ed., Addison-Wesley, 1989.

[7] L. Greengard, The Rapid Evaluation of Potential Fields in Particle Systems, The MIT Press, Cambridge, MA, 1987.

[8] F. W. Grover, Inductance Calculations: Working Formulas and Tables, Dover Publications, New York, 1946.

9] L. He and K. M. Lepak, "Simultaneous Shield Insertion and Net ordering for Capacitive and Inductive Coupling Minimization," International Symposium on Physical Design, pp. 55-60, 2000.

[10] L. He, N. Chang, S. Lin, and O. S. Nakagawa, "An Efficient Inductance Modeling for On-chip Interconnects," IEEE Custom Integrated Circuits Conference, pp. 22.6.1-22.6.4, 1999.

[11] Y. I. Ismail and E. G. Friedman, "Effects of Inductance on the Propagation Delay and Repeater Insertion in VLSI Circuits," IEEE Trans. Very Large Scale Integration (VLSI) Systems, Vol. 8, Issue: 2, April 2000

[12] M. Kamon, M. J. Tsuk, and J. K. White, "FastHenry: a Multipole-accelerated 3D Inductance Extraction Program," IEEE Trans. Computer-Aided Design, pp. 1750-1758, Sept. 1994

[13] J. R. Phillips, M. Kamon, and J. White, "An FFT-based Approach to Including Nondeal Ground Planes in a Fast 3-D Inductance Extraction Program," Proceedings of the IEEE Custom Integrated Circuits Conference, pp. 8.3.1-8.3.4, 1993.

[14] Semiconductor Industry Association, International Technology Roadmap for Semiconductors, 1999.

[15] G. Zhong, C. K. Koh, and K. Roy, "A Twisted-Bundle Layout Structure for minimizing Inductive Coupling Noise," IEEE International Conference on Computer Aided Design, pp. 406-411, 2000.

\begin{tabular}{|c|r|r||r|r|r||r|r|r|}
\hline$\left(l_{w}, d_{g}, w_{s}, w_{g}\right)(\mu \mathrm{m})$ & \multicolumn{1}{|c|}{ FastHenry } & \multicolumn{1}{|c|}{ Time $(\mathrm{sec})$} & \multicolumn{1}{c|}{ AMAS } & Time $(\mathrm{sec})$ & \multicolumn{1}{c|}{ Error $(\%)$} & \multicolumn{1}{c|}{ IAMAS } & Time (sec) & Error $(\%)$ \\
\hline$(2000,12,0.8,2)$ & $18.70280 \times 10^{-10} \mathrm{H}$ & 117.09 & $20.3658 \times 10^{-10} \mathrm{H}$ & 0.11 & 8.892 & $20.3299 \times 10^{-10} \mathrm{H}$ & 8.02 & 8.700 \\
\hline$(1000,12,0.8,2)$ & $9.99291 \times 10^{-10} \mathrm{H}$ & 103.41 & $10.1829 \times 10^{-10} \mathrm{H}$ & 0.11 & 1.901 & $10.1470 \times 10^{-10} \mathrm{H}$ & 5.82 & 1.542 \\
\hline$(500,12,0.8,2)$ & $5.14302 \times 10^{-10} \mathrm{H}$ & 106.79 & $5.0915 \times 10^{-10} \mathrm{H}$ & 0.11 & -1.003 & $5.0557 \times 10^{-10} \mathrm{H}$ & 5.72 & -1.698 \\
\hline$(10,12,0.8,2)$ & $0.07443 \times 10^{-10} \mathrm{H}$ & 98.60 & $0.1018 \times 10^{-10} \mathrm{H}$ & 0.11 & 36.806 & $0.0761 \times 10^{-10} \mathrm{H}$ & 6.15 & 2.222 \\
\hline$(1000,12,0.8,4)$ & $9.26282 \times 10^{-10} \mathrm{H}$ & 111.57 & $9.3216 \times 10^{-10} \mathrm{H}$ & 0.11 & 0.635 & $9.5280 \times 10^{-10} \mathrm{H}$ & 5.66 & 2.863 \\
\hline$(1000,12,1.6,2)$ & $9.60097 \times 10^{-10} \mathrm{H}$ & 110.11 & $8.8601 \times 10^{-10} \mathrm{H}$ & 0.11 & -7.716 & $8.8243 \times 10^{-10} \mathrm{H}$ & 5.71 & -8.090 \\
\hline$(1000,6,0.8,2)$ & $7.73106 \times 10^{-10} \mathrm{H}$ & 100.60 & $8.2411 \times 10^{-10} \mathrm{H}$ & 0.05 & 6.597 & $8.2231 \times 10^{-10} \mathrm{H}$ & 5.66 & 6.365 \\
\hline \multicolumn{2}{|l|}{ Average (absolute value) } & 106.88 & & 0.10 & 9.079 & & 6.11 & 4.497 \\
\hline
\end{tabular}

Table 3: Experimental results of our modelings, $A M A S$ and $I A M A S$, and FastHenry for self-inductances. The lengths of ground wires are 4,500 $\mu \mathrm{m}$.

\begin{tabular}{|c|c|c|c|c|c|c|c|c|c|}
\hline $\begin{array}{l}\text { Signal wire } \\
\text { length }(\mu \mathrm{m})\end{array}$ & $\begin{array}{l}\text { Overlapping } \\
\text { length }(\mu \mathrm{m})\end{array}$ & FastHenry & Time (sec) & AMAC & Time (sec) & Error (\%) & IAMAC & Time (sec) & Error $(\%)$ \\
\hline 2000 & 2000 & $17.1868 \times 10^{-10} \mathrm{H}$ & 141.67 & $17.3525 \times 10^{-10} \mathrm{H}$ & 0.11 & 0.964 & $17.2998 \times 10^{-10} \mathrm{H}$ & 6.10 & 0.657 \\
\hline 2000 & 1500 & $12.2176 \times 10^{-10} \mathrm{H}$ & 141.98 & $13.0143 \times 10^{-10} \mathrm{H}$ & 0.11 & 6.521 & $13.0140 \times 10^{-10} \mathrm{H}$ & 15.82 & 6.518 \\
\hline 1000 & 1000 & $9.2767 \times 10^{-10} \mathrm{H}$ & 114.34 & $8.6762 \times 10^{-10} \mathrm{H}$ & 0.06 & -6.473 & $8.6237 \times 10^{-10} \mathrm{H}$ & 5.66 & -7.039 \\
\hline 1000 & 500 & $4.3076 \times 10^{-10} \mathrm{H}$ & 116.70 & $4.3381 \times 10^{-10} \mathrm{H}$ & 0.05 & 0.709 & $4.3379 \times 10^{-10} \mathrm{H}$ & 5.22 & 0.705 \\
\hline 500 & 400 & $3.7163 \times 10^{-10} \mathrm{H}$ & 110.28 & $3.4705 \times 10^{-10} \mathrm{H}$ & 0.05 & -6.614 & $3.4684 \times 10^{-10} \mathrm{H}$ & 17.09 & -6.669 \\
\hline 500 & 200 & $1.7031 \times 10^{-10} \mathrm{H}$ & 110.00 & $1.7353 \times 10^{-10} \mathrm{H}$ & 0.11 & 1.887 & $1.7352 \times 10^{-10} \mathrm{H}$ & 9.12 & 1.884 \\
\hline 100 & 50 & $0.4887 \times 10^{-10} \mathrm{H}$ & 105.41 & $0.4338 \times 10^{-10} \mathrm{H}$ & 0.11 & -11.237 & $0.4321 \times 10^{-10} \mathrm{H}$ & 4.94 & -11.586 \\
\hline 100 & 25 & $0.2422 \times 10^{-10} \mathrm{H}$ & 106.55 & $0.2169 \times 10^{-10} \mathrm{H}$ & 0.06 & -10.435 & $0.2191 \times 10^{-10} \mathrm{H}$ & 11.70 & -9.523 \\
\hline 100 & 5 & $0.0547 \times 10^{-10} \mathrm{H}$ & 109.70 & $0.0434 \times 10^{-10} \mathrm{H}$ & 0.05 & -20.706 & $0.0561 \times 10^{-10} \mathrm{H}$ & 12.03 & 2.553 \\
\hline \multicolumn{3}{|c|}{ Average (absolute value) } & 117.40 & & 0.08 & 7.283 & & 9.74 & 5.683 \\
\hline
\end{tabular}

Table 4: Experimental results of our modelings, $A M A C$ and $I A M A C$, and FastHenry for coupling inductances. Here, $w_{s}=d_{s}=0.8 \mu \mathrm{m}$ for both signal wires, both ground wire length $=4,500 \mu \mathrm{m}, w_{g}=2 \mu \mathrm{m}, d_{g}=12 \mu \mathrm{m}$. 